\title{
Prediction of the Flash Point of Binary and Ternary Straight-Chain Alkane Mixtures
}

\author{
X. Li, P. P. Duan, K. N. Sun, and X. Yan \\ Beijing Key Laboratory for Chemical Power Source and Green Catalysis, School of Chemical Engineering and Environment, \\ Beijing Institute of Technology, Beijing 100081, China
}

Correspondence should be addressed to X. Li; klkxlx@163.com

Received 18 January 2014; Accepted 31 March 2014; Published 23 April 2014

Academic Editor: Bin Li

Copyright (C) $2014 \mathrm{X}$. Li et al. This is an open access article distributed under the Creative Commons Attribution License, which permits unrestricted use, distribution, and reproduction in any medium, provided the original work is properly cited.

The flash point is an important physical property used to estimate the fire hazard of a flammable liquid. To avoid the occurrence of fire or explosion, many models are used to predict the flash point; however, these models are complex, and the calculation process is cumbersome. For pure flammable substances, the research for predicting the flash point is systematic and comprehensive. For multicomponent mixtures, especially a hydrocarbon mixture, the current research is insufficient to predict the flash point. In this study, a model was developed to predict the flash point of straight-chain alkane mixtures using a simple calculation process. The pressure, activity coefficient, and other associated physicochemical parameters are not required for the calculation in the proposed model. A series of flash points of binary and ternary mixtures of straight-chain alkanes were determined. The results of the model present consistent experimental results with an average absolute deviation for the binary mixtures of $0.7 \%$ or lower and an average absolute deviation for the ternary mixtures of $1.03 \%$ or lower.

\section{Introduction}

When a flammable liquid is transported, used, or stored, safe handling is particularly important, which requires knowledge of the liquid's fire and explosion hazard. An important property used to estimate the risk of fire and explosion for a flammable liquid is the flash point $[1,2]$. When the fuel is heated, volatile combustible gases mix with air near the liquid surface. The vapor of the combustible liquid is ignited by a spark when a certain concentration is reached. The minimum temperature at this concentration is defined as the flash point.

Most flash points can be obtained by experimental analysis, but for certain rare or mixed material the flash point is difficult to obtain [3]. The typical experimental method is not only time consuming but also difficult to perform when determining the flash point for each liquid in the mixture. The experimental results are often different because of the equipment used, the environmental conditions, and the operation method chosen. In addition, although flash point values can be found in reference materials, the accuracy of the data is not guaranteed. For example, Kwon et al. [4] determined the flash point of decanol and discovered that it did not belong to the combustible materials category, where it was included on the material safety data sheet.

To compensate for the disadvantages of the experimental method, models have been proposed for calculating the flash point. The models of flash point prediction are typically based on the relationship between the flash point and the thermodynamic properties of the compound. Evaporability is determined by the boiling point, and the flash point depends on the evaporability; therefore, a good relationship is found between flash point and boiling point. Many models have been reported using the correlation between the boiling point and the flash point of pure compounds. A quadratic correlation was proposed by Patil [5] in the following equation:

$$
T_{f}=4.656+0.844 * T_{b}-0.234 * 10^{-3}\left(T_{b}\right)^{2} .
$$

In (1), $T_{f}$ (in Kelvin) is the flash point temperature and $T_{b}$ (in Kelvin) is the boiling point temperature. These values are used to calculate the flash point of organic compounds. The calculation results can reach $90 \%$ accuracy. However, studies have shown that limitations exist when this calculation is applied to silicone compounds. Wang and Sun [6] simplified 
the form of (1) and established a linear function in the following equation:

$$
T_{f}=33.176+0.67465 * T_{b} .
$$

Equation (2) can be applied to 1,457 organic compounds with an average error of $3.75 \%$. Although the form of (2) is relatively simple, the results are unreliable.

For mixtures, the relationship between the flash point and the vapor pressure of a compound is used to predict the flash point. According to the definition of flash point, a model was expressed using the following equation [7]:

$$
\mathrm{LFL}_{i}=\frac{P_{i, f}^{\delta}}{P},
$$

where $\mathrm{LFL}_{i}$ is the lower flammability limits of component $i$, $P_{i, f}^{\delta}$ (in $\left.\mathrm{kPa}\right)$ is the saturated vapor pressure, and $P$ (in $\mathrm{kPa}$ ) is the ambient pressure. The advantage of this model is that it can effectively predict the flash point of a nonideal solution.

Currently, a novel approach for predicting the flash point of alkanes employs the introduction of certain physical and chemical effects into the prediction model. For example, Valenzuela [8] presented a model that included physical and chemical effects to estimate the flash point of a pure alkane. The incorporation of a physical effect was accomplished by using the normal boiling temperature $\left(T_{b}\right)$ as an independent variable. The heat of combustion $\left(\Delta \mathrm{H}_{\mathrm{r}}\right)$ and the heat of evaporation $\left(\Delta \mathrm{H}_{\mathrm{v}}\right)$ were included as the chemical effect. The group contribution method was used to produce the following model:

$$
\begin{aligned}
\frac{T_{f}}{T_{b}}= & \beta_{0}+\beta_{1}\left(\frac{\Delta \mathrm{H}_{\mathrm{r}}}{\Delta \mathrm{H}_{\mathrm{v}}}\right) \\
& +\alpha_{1} \sum n_{1}+\alpha_{2} \sum n_{2}+\alpha_{3} \sum n_{4}+\alpha_{5} \sum n_{5},
\end{aligned}
$$

where $\beta_{0}, \beta_{1}, \alpha$, and $n$ are defined values that are specific to the different alkanes. Similarly, Mathieu [9] studied the quantitative structure-property relationship [10] to predicate the flash point of alkanes. This group developed a method based on the backpropagation ANN model using the group bond contribution method. In addition to considering physical and chemical properties, Li developed a relatively simple model to calculate the flash point using the following equation [11]:

$$
\operatorname{Lg}\left(A-T_{f}\right)=B-C N^{2 / 3},
$$

where $A, B$, and $C$ are constants and $N$ is the number of organic carbon atoms. Equation (5) can be applied to straightchain saturated alkanes and straight-chain monoolefins. The calculated results and the literature results for these types of compounds are in good agreement. The error is small, and the average absolute deviation is not more than $1.5 \%$. However, there are three constants in (5), and different substances have different constants, which makes the use of this calculation cumbersome.

Therefore, these models can be applied to pure substances or substances with known thermodynamic information.
However, certain model results are not accurate, and the error is large. The other models are complicated or include many parameters and, therefore, are inconvenient for practical use. The largest disadvantage in using these models is that most cannot predict the flash point of multivariate alkane mixtures. Thus, limitations for the flash point prediction of alkane mixtures still exist. To predict the flash point of straightchain alkane mixtures, a model was developed to reduce the number of required experiments. In this model, the thermodynamic parameters are not required.

\section{Model}

The model for straight-chain alkane mixtures was proposed for predicting flash point using the following equation:

$$
\frac{1}{T}=\sum_{i=1}^{n} \frac{x_{i}}{T_{i}}+\sum_{i=1}^{n} \sum_{j>i}^{n} \frac{K_{i j} x_{i} x_{j}}{\sqrt{T_{i} T_{j}}} .
$$

In the model, coefficient $K_{i j}$ was determined using experimental values. The specific calculation method is as follows.

(1) Any two straight-chain alkanes are chosen to determine the flash points, $T_{i}$ and $T_{j}$.

(2) The chosen alkanes are mixed according to a volume ratio of $1: 1$, and the flash point $T$ is determined.

(3) $K_{i j}$ is calculated using the determined results and the model.

\section{Experiment}

To calculate the coefficient in the modified model, certain flash points were determined experimentally. The reagents and instruments used are listed in Table 1.

Flash point determination methods are typically classified as either a closed cup tester or an open cup tester. In this study, the WFY-111C flash point tester is used to determine the flash point, which is a closed cup tester. When the flash point of the compound is expected to be less than $50^{\circ} \mathrm{C}$, a heating rate of $1^{\circ} \mathrm{C} / \mathrm{min}$ is required while stirring the solution. The ignition test begins when the solution temperature is $10^{\circ} \mathrm{C}$ lower than the expected flash point value. When the flash point of the compound is expected to be higher than $50^{\circ} \mathrm{C}$, the temperature is allowed to rise in $2-3^{\circ} \mathrm{C}$ per minute while stirring the solution. The ignition test begins when the solution temperature is $10^{\circ} \mathrm{C}$ lower than the expected flash point value. The lowest temperature at which the vapor above the sample surface will briefly ignite is the flash point. Each experiment was performed in triplicate.

\section{Results and Discussion}

4.1. The Determination of Flash Point. A selection of pure alkanes and straight-chain alkane mixtures were chosen for the experiment. The flash points of these compounds are shown in Tables 2 and 3. 
TABLE 1: Reagents and instruments.

\begin{tabular}{lccc}
\hline Name & Standard & Content & Manufacturer \\
\hline Octane & AR & $99 \%$ & Tianjin Fu Chen Chemical Reagent \\
Nonane & AR & $99 \%$ & Tianjin Guangfu Institute of Fine Chemicals \\
Decane & AR & $99 \%$ & Shanghai Aladdin Chemical Reagent \\
Undecane & AR & $99 \%$ & Shanghai Aladdin Chemical Reagent \\
Dodecane & AR & $99 \%$ & Shanghai Aladdin Chemical Reagent \\
Flash point tester & WFY-111C & Dalian Wuzhou Oil Equipment \\
\hline
\end{tabular}

TABLE 2: Flash points of the pure alkanes.

\begin{tabular}{lc}
\hline Pure alkanes & Flash point $\left({ }^{\circ} \mathrm{C}\right)$ \\
\hline Octane & 18.5 \\
Nonane & 35.5 \\
Decane & 53.0 \\
Undecane & 65.0 \\
Dodecane & 81.0 \\
\hline
\end{tabular}

TABLE 3: Flash points of the straight-chain alkane mixtures using the volume ratio $1: 1$.

\begin{tabular}{lc}
\hline Straight-chain alkane mixtures & Flash point $\left({ }^{\circ} \mathrm{C}\right)$ \\
\hline Nonane + decane & 41.0 \\
Octane + dodecane & 27.0 \\
Nonane + dodecane & 44.0 \\
Octane + decane & 26.0 \\
Decane + dodecane & 63.0 \\
Nonane + undecane & 43.0 \\
Decane + undecane & 59.0 \\
\hline
\end{tabular}

TABLE 4: The coefficient $K_{i j}$.

\begin{tabular}{lc}
\hline Straight-chain alkane mixtures & Coefficient $K_{i j}$ \\
\hline Nonane + decane & -0.0732 \\
Octane + dodecane & -0.1511 \\
Nonane + dodecane & 0.0896 \\
Octane + decane & 0.0743 \\
Decane + dodecane & 0.0014 \\
Nonane + undecane & 0.1388 \\
Decane + undecane & -0.0748 \\
\hline
\end{tabular}

TABLE 5: Flash point of octane + dodecane with different mole fractions.

\begin{tabular}{lcccc}
\hline \multicolumn{3}{c}{ Mole fraction } & \multicolumn{3}{c}{ Flash point, $T_{f},{ }^{\circ} \mathrm{C}$} & $\left|\Delta T_{f}\right|,{ }^{\circ} \mathrm{C}$ \\
Octane & Dodecane & Exp. & Model & \\
\hline 0.00 & 1.00 & 81.00 & 81.00 & 0.00 \\
0.31 & 0.69 & 42.00 & 39.62 & 2.38 \\
0.54 & 0.46 & 29.50 & 28.56 & 0.94 \\
0.73 & 0.27 & 23.20 & 23.33 & 0.13 \\
0.88 & 0.12 & 21.20 & 20.46 & 0.74 \\
1.00 & 0.00 & 18.50 & 18.50 & 0.00 \\
\hline \multicolumn{5}{c}{ Average absolute deviation, ${ }^{\circ} \mathrm{C}$} \\
\hline
\end{tabular}

TABle 6: Flash point of nonane + decane with different mole fractions.

\begin{tabular}{|c|c|c|c|c|}
\hline \multicolumn{2}{|c|}{ Mole fraction } & \multicolumn{2}{|c|}{ Flash point, $T_{f},{ }^{\circ} \mathrm{C}$} & \multirow{2}{*}{$\left|\Delta T_{f}\right|,{ }^{\circ} \mathrm{C}$} \\
\hline Nonane & Decane & Exp. & Model & \\
\hline 0.00 & 1.00 & 53.00 & 53.00 & 0.00 \\
\hline 0.22 & 0.78 & 48.50 & 47.71 & 0.79 \\
\hline 0.46 & 0.54 & 44.00 & 43.18 & 0.82 \\
\hline 0.65 & 0.35 & 39.80 & 40.14 & 0.34 \\
\hline 0.83 & 0.17 & 37.50 & 37.58 & 0.08 \\
\hline 1.00 & 0.00 & 35.50 & 35.50 & 0.00 \\
\hline \multicolumn{4}{|c|}{ Average absolute deviation, ${ }^{\circ} \mathrm{C}$} & 0.34 \\
\hline
\end{tabular}

TABLE 7: Flash point of nonane + dodecane with different mole fractions.

\begin{tabular}{|c|c|c|c|c|}
\hline \multicolumn{2}{|c|}{ Mole fraction } & \multicolumn{2}{|c|}{ Flash point, $T_{f},{ }^{\circ} \mathrm{C}$} & \multirow{2}{*}{$\left|\Delta T_{f}\right|,{ }^{\circ} \mathrm{C}$} \\
\hline Nonane & Dodecane & Exp. & Model & \\
\hline 0.00 & 1.00 & 81.00 & 81.00 & 0.00 \\
\hline 0.28 & 0.72 & 58.80 & 59.71 & 0.91 \\
\hline 0.51 & 0.49 & 48.00 & 48.99 & 0.99 \\
\hline 0.70 & 0.30 & 41.50 & 42.60 & 1.10 \\
\hline 0.87 & 0.13 & 37.80 & 38.41 & 0.61 \\
\hline 1.00 & 0.00 & 35.50 & 35.50 & 0.00 \\
\hline \multicolumn{4}{|c|}{ Average absolute deviation, ${ }^{\circ} \mathrm{C}$} & 0.60 \\
\hline
\end{tabular}

TABLE 8: Flash point of octane + decane + dodecane with different mole fractions.

\begin{tabular}{lccccc}
\hline & Mole fraction & \multicolumn{3}{c}{ Flash point, $T_{f},{ }^{\circ} \mathrm{C}$} & \multirow{2}{*}{$\left|\Delta T_{f}\right|,{ }^{\circ} \mathrm{C}$} \\
Octane & Decane & Dodecane & Exp. & Model & \\
\hline 0.00 & 0.00 & 1.00 & 81.00 & 81.00 & 0.00 \\
0.00 & 1.00 & 0.00 & 53.00 & 53.00 & 0.00 \\
0.21 & 0.17 & 0.62 & 47.50 & 44.99 & 2.51 \\
0.20 & 0.65 & 0.15 & 42.50 & 40.17 & 2.33 \\
0.39 & 0.32 & 0.29 & 31.50 & 32.65 & 1.15 \\
0.71 & 0.15 & 0.13 & 22.00 & 23.20 & 1.20 \\
1.00 & 0.00 & 0.00 & 18.50 & 18.50 & 0.00 \\
\hline \multicolumn{5}{c}{ Average absolute deviation, ${ }^{\circ} \mathrm{C}$} \\
\hline
\end{tabular}

4.2. The Calculation Results of the Coefficient $K_{i j}$. In the model, the coefficient $K_{i j}$ can be calculated using the flash point of the pure alkanes and any binary alkane mixture. Different mixtures have different coefficients. There are seven 
TABLE 9: Flash point of nonane + decane + undecane with different mole fractions

\begin{tabular}{lccccc}
\hline \multicolumn{3}{c}{ Mole fraction } & \multicolumn{3}{c}{ Flash point, $T_{f},{ }^{\circ} \mathrm{C}$} \\
Nonane & Decane & Undecane & Exp. & Model & $\left|\Delta T_{f}\right|,{ }^{\circ} \mathrm{C}$ \\
\hline 0.00 & 0.00 & 1.00 & 65.00 & 65.00 & 0.00 \\
0.19 & 0.17 & 0.63 & 54.50 & 54.26 & 0.24 \\
0.00 & 1.00 & 0.00 & 53.00 & 53.00 & 0.00 \\
0.18 & 0.66 & 0.15 & 49.50 & 49.92 & 0.42 \\
0.36 & 0.33 & 0.31 & 46.50 & 47.24 & 0.74 \\
0.69 & 0.16 & 0.15 & 39.50 & 40.36 & 0.86 \\
1.00 & 0.00 & 0.00 & 35.50 & 35.50 & 0.00 \\
\hline \multicolumn{5}{c}{ Average absolute deviation, ${ }^{\circ} \mathrm{C}$} \\
\hline \multicolumn{5}{c}{}
\end{tabular}

TABLE 10: Flash point of nonane + decane + dodecane with different mole fractions.

\begin{tabular}{lccccc}
\hline \multicolumn{3}{c}{ Mole fraction } & \multicolumn{3}{c}{ Flash point, $T_{f},{ }^{\circ} \mathrm{C}$} \\
Nonane & Decane & Dodecane & Exp. & Model & $\left|\Delta T_{f}\right|,{ }^{\circ} \mathrm{C}$ \\
\hline 0.00 & 0.00 & 1.00 & 81.00 & 81.00 & 0.00 \\
0.19 & 0.18 & 0.63 & 58.40 & 60.30 & 1.90 \\
0.00 & 1.00 & 0.00 & 53.00 & 53.00 & 0.00 \\
0.18 & 0.67 & 0.15 & 50.50 & 51.03 & 0.53 \\
0.37 & 0.34 & 0.30 & 47.50 & 49.22 & 1.72 \\
0.69 & 0.16 & 0.14 & 38.50 & 41.01 & 2.51 \\
1.00 & 0.00 & 0.00 & 35.50 & 35.50 & 0.00 \\
\hline \multicolumn{5}{c}{ Average absolute deviation, ${ }^{\circ} \mathrm{C}$} \\
\hline
\end{tabular}

mixtures used in this study, and their flash points are listed in Tables 2 and 3. The calculation results are shown in Table 4.

4.3. The Prediction of the Flash Point for Binary Alkane Mixtures. Three groups of binary alkane mixtures were selected for flash point determination. According to the model, the flash point of these mixtures can be obtained from the calculation. The results are shown in Tables 5, 6, and 7.

The results show that there is a good agreement between the experimental results and the calculated results. The maximum absolute deviation between the model and the experimental results is $1.10^{\circ} \mathrm{C}$. The average absolute deviation is $0.60^{\circ} \mathrm{C}$. The model calculation results are closer to the experimental results than those achieved by other models, and, therefore, this model has better predictability and applicability.

Three graphs are shown in Figures 1, 2, and 3 to visually compare the results.

The figures show that the calculated results using the model have a high consistency with the experimental results. Although the model contains multiple parameters, the calculation process is relatively straightforward and the calculated results have a high accuracy. In the next section, experimental determinations of the flash point of ternary alkane mixtures are used to verify the model.

4.4. The Prediction of the Flash Point of Ternary Alkane Mixtures. Similar to the binary alkane mixtures, three groups

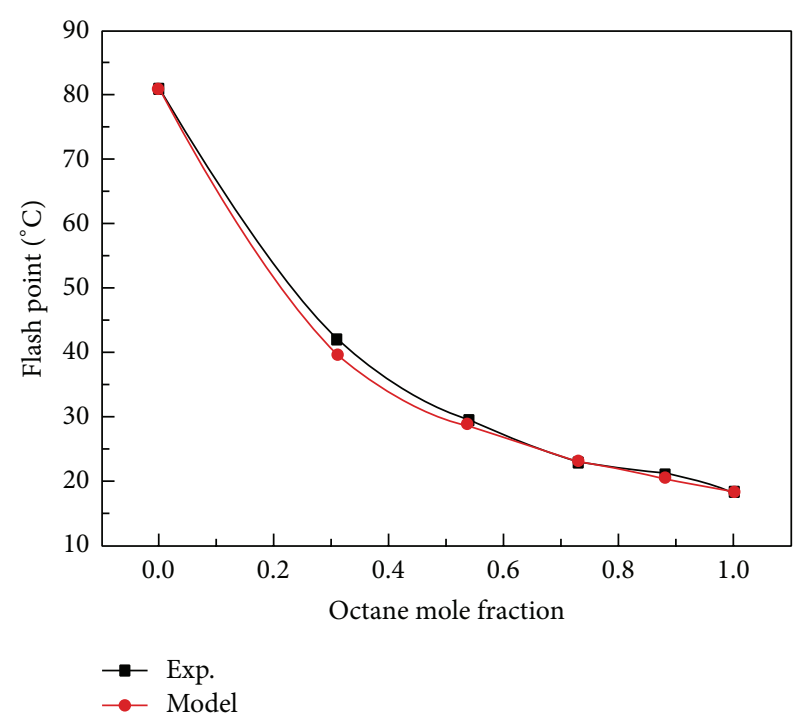

FIGURE 1: Flash point of octane + dodecane with different mole fractions.

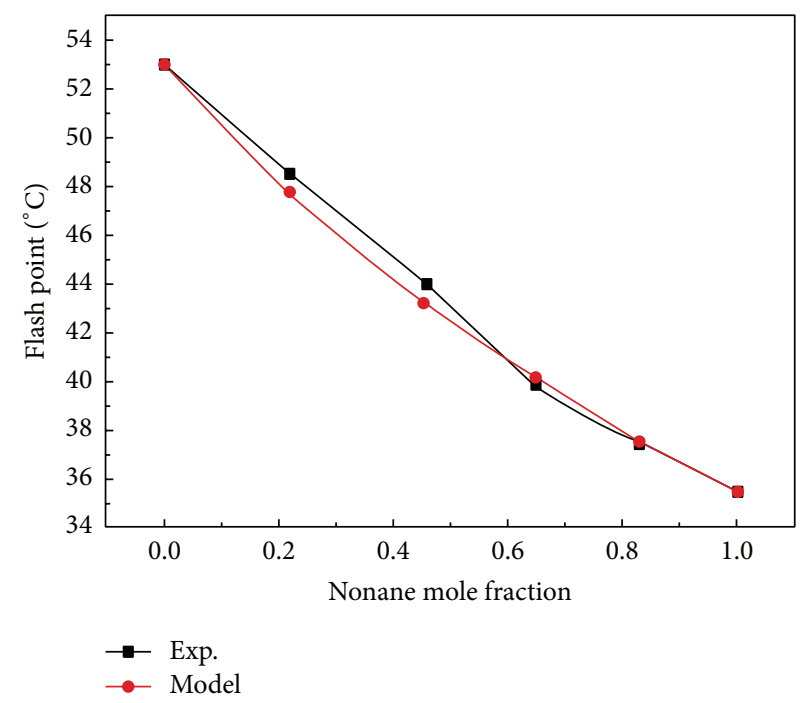

FIGURE 2: Flash point of nonane + decane with different mole fractions.

of ternary alkane mixtures were selected to determine the experimental flash point values. According to the developed models, the flash point can also be obtained using calculations. The results are shown in Tables 8, 9, and 10 .

The maximum absolute deviation between the model and the experimental results is $2.51^{\circ} \mathrm{C}$. The average absolute deviation is $1.03^{\circ} \mathrm{C}, 0.32^{\circ} \mathrm{C}$, and $0.95^{\circ} \mathrm{C}$ for the nonane, decane, and dodecane mixtures, respectively. The model calculation results are in a good agreement with the experimental results, and, therefore, the model has better predictability and applicability than other flash point models. 


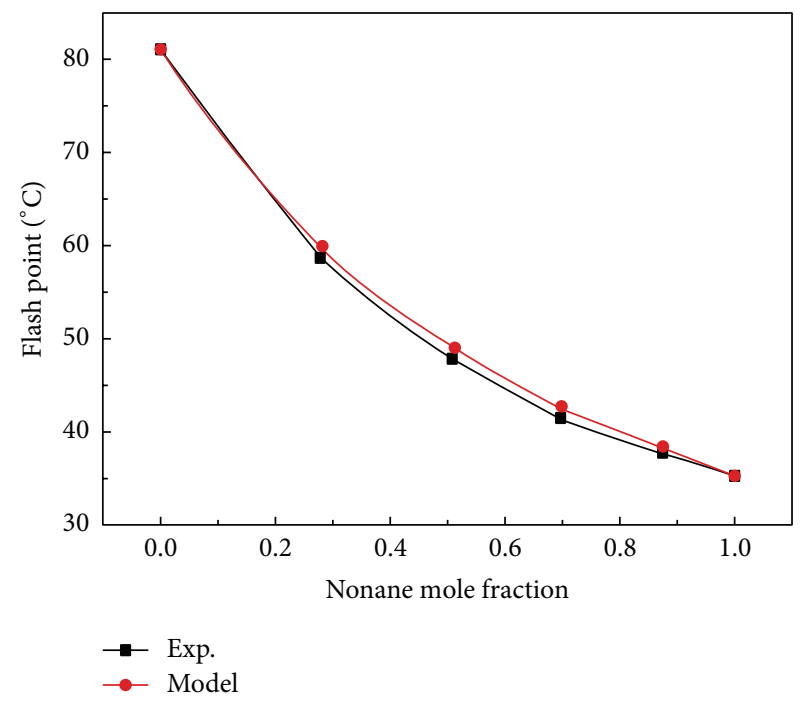

Figure 3: Flash point of nonane + dodecane with different mole fractions.

\section{Conclusion}

A model for predicting the flash point of multiple mixtures of straight-chain alkanes was established in this study. Compared with the experimental results, the model presents good applicability. In addition, the model describes a simple calculation process with highly accurate results. Therefore, the model is more suitable than previously proposed models for the calculation of the flash point of alkanes and their mixtures.

\section{Symbols}

$V_{i}$ : A volume fraction of component $i$ in the mixture, $\%$

$T$ : The flash point of binary or ternary straight-chain alkane mixtures, ${ }^{\circ} \mathrm{C}$

$T_{i}$ : The flash point of pure component $i,{ }^{\circ} \mathrm{C}$

$x_{i}$ : A mole fraction of component $i$ in the mixture, $\%$

$K$ : A dimensionless number.

\section{Conflict of Interests}

The authors declare that there is no conflict of interests regarding the publication of this paper.

\section{Acknowledgments}

This study was supported by the International Science and Technology Cooperation Program of China (no. 2012DFR40240s) and the Beijing Key Laboratory for Chemical Power Source and Green Catalysis under Contract no. 2013CX02031.

\section{References}

[1] H. J. Liaw and Y. Y. Chiu, "A general model for predicting the flash point of miscible mixtures," Journal of Hazardous Materials, vol. 137, no. 1, pp. 38-46, 2006.

[2] H. J. Liaw, V. Gerbaud, and H. T. Wu, "Flash-point measurements and modeling for ternary partially miscible aqueousorganic mixtures," Journal of Chemical and Engineering Data, vol. 55, no. 9, pp. 3451-3461, 2010.

[3] S. Y. Kim and B. Lee, "A prediction model for the flash point of binary liquid mixtures," Journal of Loss Prevention in the Process Industries, vol. 23, no. 1, pp. 166-169, 2010.

[4] K. Kwon, D. Lee, Y. Iwata, and H. Koseki, "Flash points of ndecanol," Journal of Loss Prevention in the Process Industries, vol. 21, no. 4, pp. 478-480, 2008.

[5] G. S. Patil, "Estimation of flash point," Fire and Materials, vol. 12, no. 3, pp. 127-131, 1988.

[6] K. Q. Wang and X. Z. Sun, "Correlation and calculation of the flash points of organic compounds," Computational Applied Chemistry, vol. 18, pp. 581-584, 2001.

[7] X. S. Liu and Z. Y. Liu, "Research progress on flash point prediction," Journal of Chemical and Engineering Data, vol. 55, no. 9, pp. 2943-2950, 2010.

[8] E. M. Valenzuela, R. Vázquez-Román, S. Patel, and M. S. Mannan, "Prediction models for the flash point of pure components," Journal of Loss Prevention in the Process Industries, vol. 24, no. 6, pp. 753-757, 2011.

[9] D. Mathieu, "Inductive modeling of physico-chemical properties: flash point of alkanes," Journal of Hazardous Materials, vol. 179, no. 1-3, pp. 1161-1164, 2010.

[10] Y. Pan, J. Jiang, and Z. Wang, "Quantitative structure-property relationship studies for predicting flash points of alkanes using group bond contribution method with back-propagation neural network," Journal of Hazardous Materials, vol. 147, no. 1-2, pp. 424-430, 2007.

[11] H. J. Li, "Discussion on calculation methods of organic flash point," Safety, Health and Environment, vol. 10, pp. 40-42, 2010. 

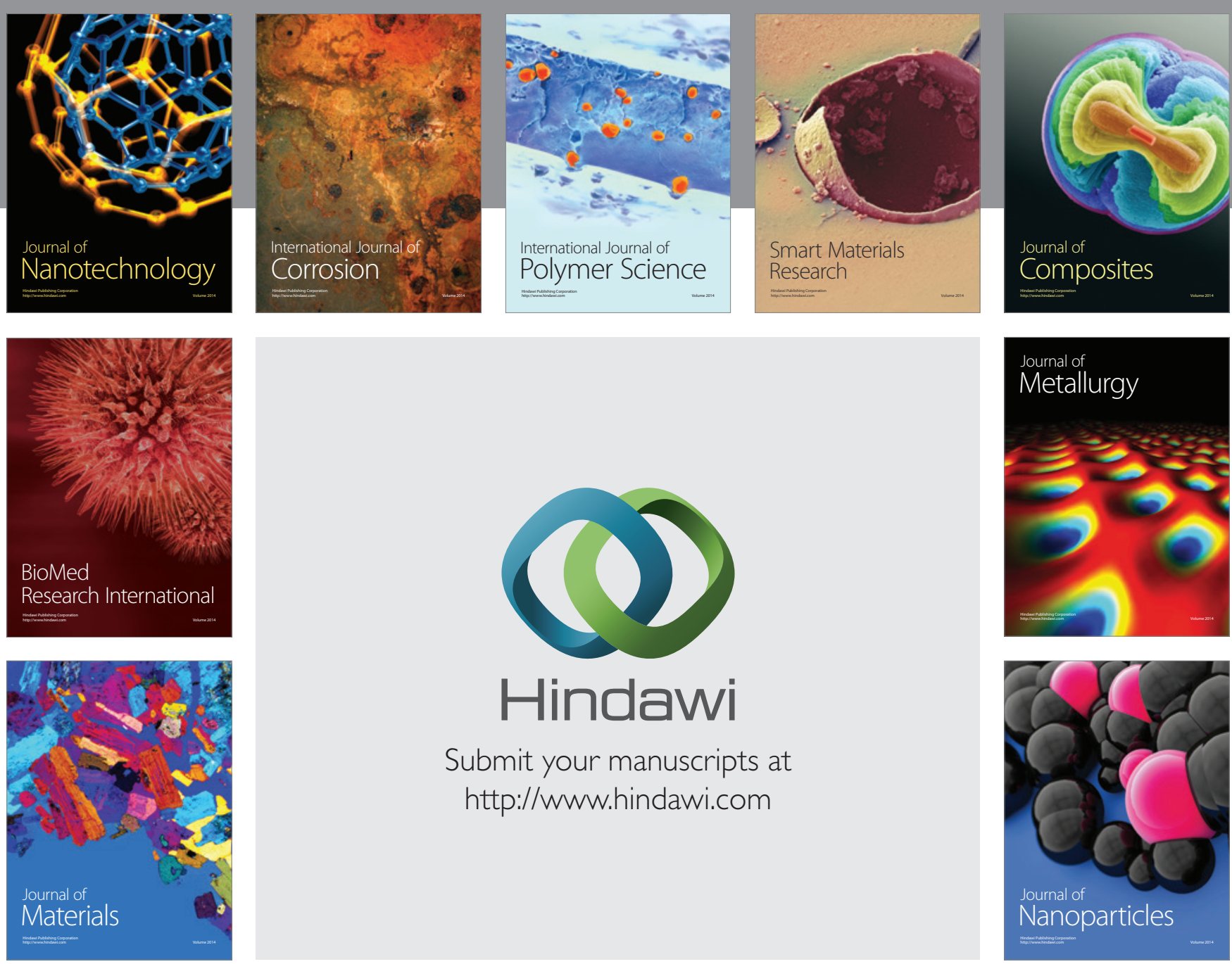

Submit your manuscripts at http://www.hindawi.com
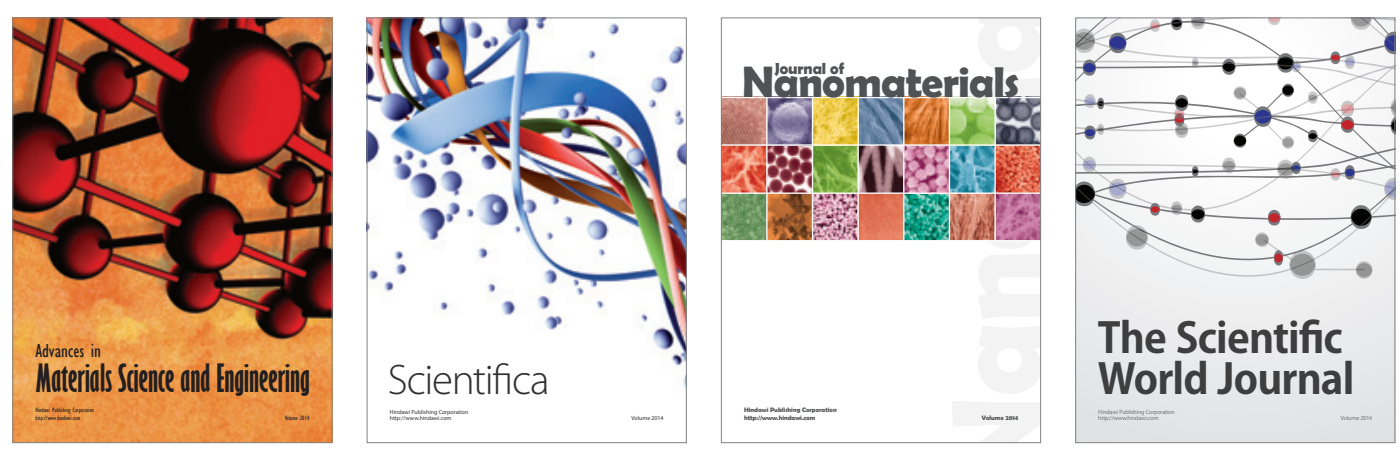

\section{The Scientific World Journal}
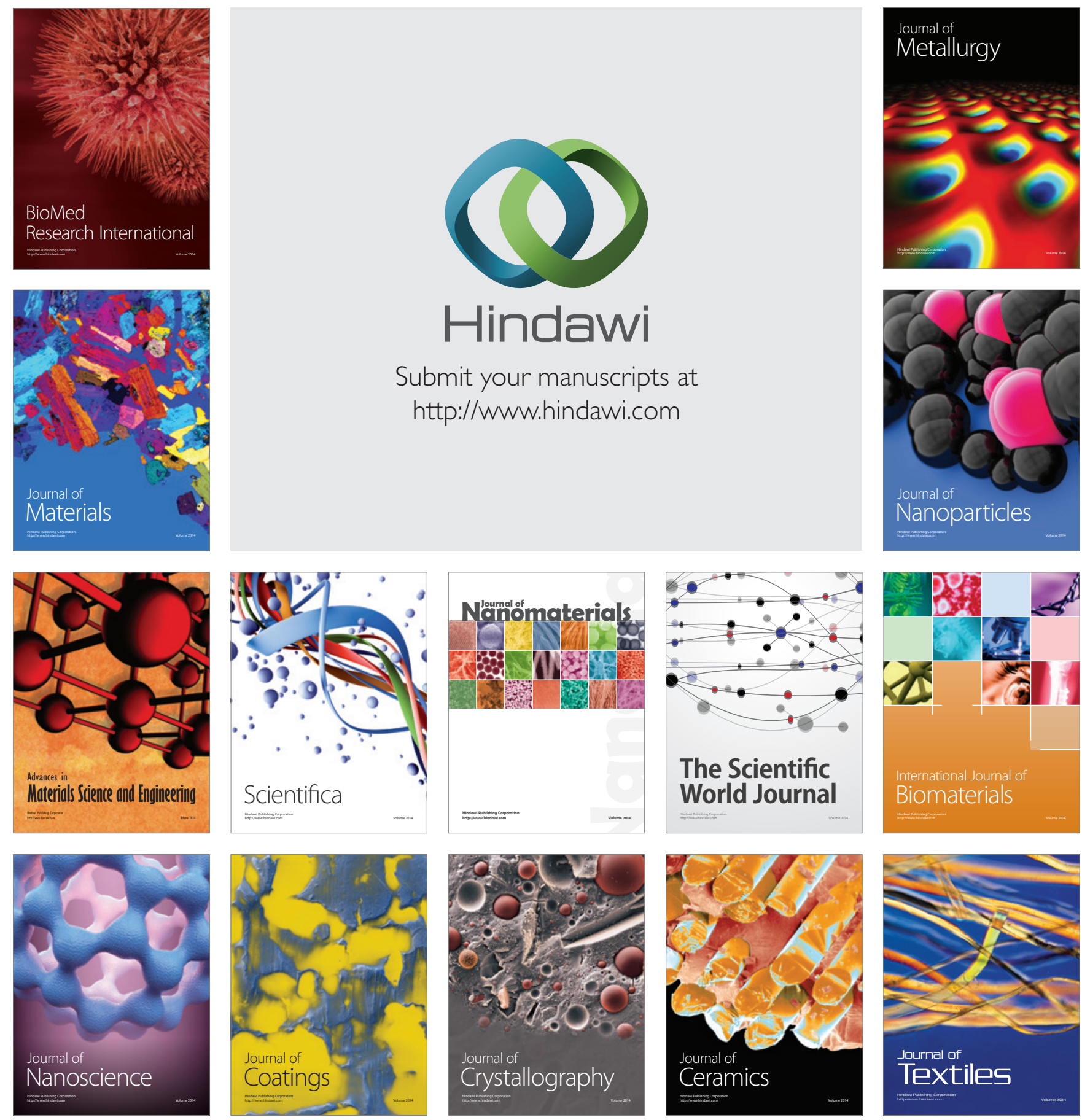